\title{
NONLINEAR ALFVÉN WAVES WITH LARGE LARMOR RADIUS EFFECT
}

\author{
NAGENDRA KUMAR and KRISHNA M. SRIVASTAVA \\ Department of Mathematics \\ University of Roorkee \\ Roorkee 247667 INDIA
}

The nonlinear behaviour of Alfvén waves propagating parallel to a uniform magnetic field in a compressible fluid with large Larmor radius effect is investigated. We have considered an inviscid nonheat conducting gas of infinite electrical conductivity with scalelength of interest $L$ such that $L<<\rho_{i}$; where $\rho_{i}$ is the ion gyroradius. Its motion is governed by basic MHD equations. A reductive perturbation analysis, including weak spatial variation in the transverse direction, based upon the coordinate stretchings $\xi=\epsilon(x-\lambda t), n=\epsilon^{3 / 2} y, \zeta=\epsilon^{3 / 2} z, \tau=\epsilon^{2} t$ is performed. The transverse components of magnetic field and velocity, denoted by means of the complex quantities $\tilde{V}\left(=V_{y}+i V_{z}\right)$ and $\widetilde{B}\left(=B_{y}+i B_{z}\right)$, have expansions of the form $\widetilde{V}=\epsilon^{1 / 2}\left(\tilde{V}^{(1)}+\epsilon \tilde{V}^{(2)}+\ldots\right)$ and $\left.\tilde{B}=\epsilon^{1 / 2}\left(\tilde{B}^{1}\right)+\epsilon \widetilde{B}\left({ }^{2}\right)+\ldots\right)$, whil $\epsilon$ the other variables have expansions $B_{X}=B_{O}+\epsilon B_{x}\left({ }^{1}\right)+\ldots, V_{X}=\epsilon V_{X}\left({ }^{1}\right)_{+}$ $\epsilon^{2} V_{x}\left({ }^{2}\right)+\ldots, \rho=\rho_{0}+\epsilon \rho^{(1)}+\ldots, \quad p=p_{0}+\epsilon_{p}(1)+\ldots$. . Using the above stretchings and expansions, we obtain the set of equations governing the evolution of the transverse $\tilde{B}$ and parallel $B_{x}$ magnetic field perturbations. If the spatial variation in the transverse direction is negligible, then we get the "Derivative Nonlinear Schrödinger (DNLS) equation"

$$
\frac{\partial \widetilde{B}}{\partial \tau}+C_{2} \frac{\partial}{\partial \xi}\left(|\tilde{B}|^{2} \tilde{B}\right)+i C_{1} \frac{\partial^{2} \tilde{B}}{\partial \xi^{2}}=0 ; C_{2}=v_{A}^{3} /\left(4 B_{o}^{2}\left(v_{A}^{2}-\alpha^{2}\right)\right)
$$

The solution of the DNLS equation and its properties have been discussed by many researchers. When $C_{1}\left(=\operatorname{Mcv}_{A}^{2} / 2 \mathrm{eB}_{0}\right)=0$, the dispersive term in the DNLS equation vanishes and higher-;rder dispersive effects are required to contain the nonlinear growth of the wave. It means that the LLR effect provides a dispersive term to balance the nonlinear growth of the Alfvèn wave. It is conjectured that the present calculations may be applicable to the study of the structure of $\mathrm{MHD}$ waves in both laboratory and space plasmas e.g. plasma flow near small planetary bodies such as comets, plasma dynamics near collisionless shock fronts.

Reference:

Mjфlhus, E. and Wyller, J.(1988) 'Nonlinear Alfvèn waves in a finitebeta plasma', J. Plasma Phys. 40, 299-318. 